Original Research Paper

\title{
The Rotation Effect on Shear Horizontal Surface Wave in a Piezoelectric Semi-Space Covered by a Semiconductor Film
}

\author{
Fatimah Alshaikh \\ Department of Mathematics, Faculty of Science, Jazan University, Jazan 2097, Saudi Arabia
}

Article history

Received: 24-02-2017

Revised: 13-03-2017

Accepted: 25-04-2017

Email: falshaikh@yahoo.com

\begin{abstract}
In this study, a mathematical model of the rotation effect on Shear Horizontally wave (SH-wave) propagation in a piezoelectric semispace covered by a semiconductor film is investigated. The semiconducting layer is rotating with a constant angular velocity and the interface between the piezoelectric substrate and the semiconductor layer is imperfectly bonded. Furthermore, the surfaces of the bilayer system assumed free of traction and electrically shorted or open. The governing equations of the dynamical displacement and electrical potential function under the effect of rotation are driven by solving the coupled electromechanical field equations of the piezoelectric half-space and the semiconductor film. In addition, the exact frequency equations of $\mathrm{SH}$ waves are derived. Next, the numerical examples are considered to clarify the effects of rotation and electromagnetic boundary conditions for the different values of the film thickness and wave number on the dispersion behaviors. Finally, the effect of rotation on the frequency equation is investigated in detail for piezoelectric material PZT-5H and the semiconductor silicon. The obtained results provide a predictable and theoretical basis for applications of piezoelectric and semiconductor structures to surface acoustic wave equipments.
\end{abstract}

Keywords: SH-Waves, Effect of Rotation, Piezoelectric Materials, Semiconductor, Frequency Equation

\section{Introduction}

Piezoelectric ceramic has been effectively utilized to make different electromechanical functional systems and electronic industry devices. It is widely applied in many technological equipments like resonators, actuators, resonators, filters, sensors, delay lines, oscillators and others Yang (2008). Both Surface Acoustic Waves (SAW) and Bulk Acoustic Waves (BAW) have been applied for the acousto-electric instruments. Moreover, waveguide acoustic Shear Horizontal (SH) eigenmodes in piezoelectric layer-substrate structures have manifold employments in various electronic industry appliances, see for example, (Topolov and Bowen, 2008; Ye, 2008; Royer and Dieulesaint, 2000; Abd-Alla, 1999). furthermore, useful mathematical modeling about linear and nonlinear magnetoacoustic and thermomagnetoelastic equations are obtained by Abd-Alla and Maugin (1987; 1990a).

A wave propagating in a piezoelectric material is usually followed by producing an electric charge. In addition, if the material is additionally semiconducting, then the electric charge generates currents and space charge arising from dissipation effect and acoustic lack in piezoelectric surface waves (Hutson and White, 1962; Abd-Alla et al., 2016). The rotation effects on velocity wave provide information for the design of resonators, sensors and oscillators. Specially, velocity interchanges because of rotation have been applied to make gyroscopes (Wren and Budness, 1987; Tiersten et al., 1980). The problem of SH waves were considered in piezoelectric materials with surface layer covered by metal in Kielczynski et al. (1989). Concerning the investigation of the phenomena of the propagation of linear and nonlinear surface acoustic waves on magnetostrictive materials are considered by Abd-Alla and Maugin (1990b).

Recently, the propagation methodology of SH waves in a two different coupled structures for examples, piezoelectric-piezomagnetic, piezoelectric- elastic, piezoelectric-semiconductor and piezoelectricpolymeric are considered for various hypotheses by many authors like, Liu et al. (2010; Son and Kang, 2011; Piliposian et al., 2012; Vashishth and Dahiya, 2013; 
Zakharenko, 2013). The propagation of SH surface acoustic waves like the Bleustein-Gulyaev wave (Abd-Alla and Asker, 2016; Gua and Jin, 2015) and Rayleigh waves Abd-Alla and Alshaikh (2016), have exceptional significance for the design electronic and communication systems throughout the world.

In this study, a mathematical model has been built to investigate the influence of the rotation on the propagation characteristic of $\mathrm{SH}$ waves. We assumed that $\mathrm{SH}$ waves are propagating in a structure which contain of a piezoelectric material covered by a semiconductor film. The governing equations of the displacements and electrical field are given by finding a solution to the associated electroacoustic field equations of the piezoelectric half-space carrying the semiconductor film. In addition, the exact dispersion equations are derived. Next, the numerical examples are given to clarify the effects of the boundary conditions for the different values of the film thickness and wave number on the dispersion behaviors. Finally, the effect of the rotation on the frequency equation are investigated in detail for PZT-5H piezoelectric crystal and semiconductor silicon.

\section{Formulation of the Problem}

Assume a piezoelectric half-space substrate which considered a homogeneous transversely isotropic whose surface is carrying a semiconductor plate of thickness $2 \mathrm{~h}$ that is supposed a non piezoelectric elastic material as presented in Fig. 1. We take into account the coordinate system $o x_{1} x_{2} x_{3}$ which its origin is considered at any point on the plane surface while the axis $x_{2}$ is indicating vertically downward into the piezoelectric substrate. Therefore, the piezoelectric half-space is represented by $x_{3}$-direction and the thin layer semiconductor takes the region $0 \leq x_{3}$ $\leq-2 h$. Selecting $x_{2}$-axis as company to the direction of wave propagation such that all particles on the line parallel to the axis $x_{1}$ are evenly displaced. Thus, all the quantities are not dependent on the axis $x_{3}$.

Furthermore, consider the semiconductor plate of thickness $2 \mathrm{~h}$ is rotating regularly with an angular velocity $\vec{\Omega}=\Omega \vec{n}$, where $\vec{n}$ is the unit vector illustrating the orientation of the rotated axis. The dynamical equation of motion with the presence of rotation effect has two additional terms. The first one due to time-varying motion only which so-called centripetal acceleration and may be written as: $\vec{\Omega} \times(\vec{\Omega} \times \vec{n})$, due to time-varying motion only. The other one is the acceleration of Coriolis that may be written as: $2 \vec{\Omega} \times \vec{n}$, where $\vec{n}$ is the vector of mechanical displacement. These terms do not appear in nonrotating media (Yang, 2008).

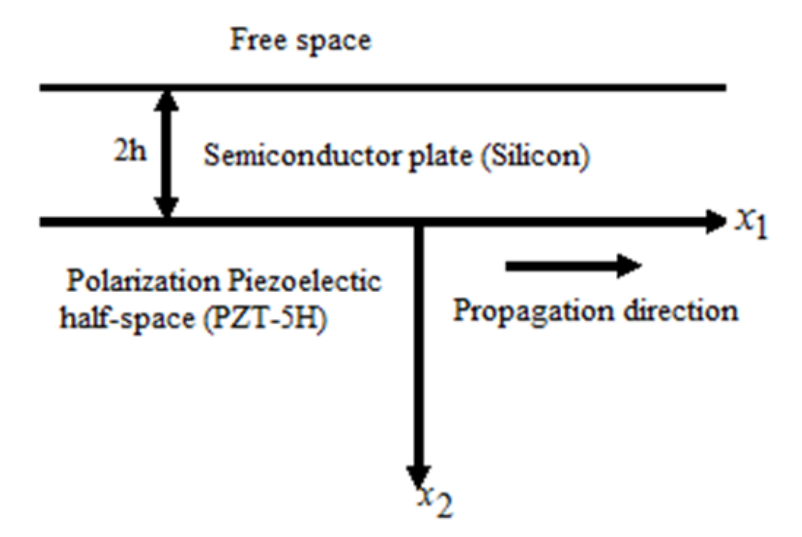

Fig. 1. A piezoelectric semi-space covered with a semiconductor film

\section{Basic Governing Equations}

The field equations of motion in semiconductor elastic layer in a rotating frame of reference are given as:

$$
\begin{gathered}
T_{i j, j}=\rho\left(\ddot{u}_{i}+\varepsilon_{i m n} \varepsilon_{m j k} \Omega_{n} \Omega_{j} u_{k}+2 \varepsilon_{i j k} \Omega_{j} \dot{u}_{k}\right), \\
D_{i, i}=q n, \quad q \dot{n}=J_{i, i}, \quad i, j=1,2,3 .
\end{gathered}
$$

where the electric current $J_{i}$ can be written as:

$$
J_{i}=q \bar{n} \mu_{i j} E_{j}+q n \mu_{i j} \bar{E}_{j}-q d_{i j} N_{j}, \quad N_{j}=n_{, j}
$$

The constitutive relations and the field equations of motion in a transversely isotropic piezoelectric $(6 \mathrm{~mm}$ class) medium in the absence of body forces and Gauss's law of electrostatics neglecting the free charge may be summarized as (Abd-Alla et al., 2016):

$$
\begin{aligned}
& \tau_{i j}=c_{i j k l} S_{k l}-e_{k i j} E_{k}, \quad D_{i}=e_{i j k} S_{j k}+\varepsilon_{i j} E_{j} . \\
& S_{i j}=\frac{1}{2}\left(u_{i, j}+u_{j, i}\right), \quad E_{i}=-\varphi_{, i} \\
& \tau_{i j, j}=\rho \ddot{u}_{i}, \quad D_{i, i}=0, \quad i, j=1,2,3 .
\end{aligned}
$$

\section{Equations for a Semiconductor Film}

Assume a semiconductor film of thickness $2 h$, which is considered very thin. We are concerned with a thin layer, as illustrated in Fig. 1. So, the components of stress may be taken to vanish, i.e.:

$T_{2 j}=0, \quad j=1,2,3$.

Corresponding to the consolidated notation of matrix (Abd-Alla and Asker, 2016), where $p, q$ as $1,2,3, \ldots$. and 6 , then the Equation 6 becomes: 


$$
T_{q}=0, \quad q=2,4,6
$$

As well as the constitutive relations (3) may be written as:

$$
T_{r}=\bar{c}_{r s} S_{s}-\bar{e}_{k r} E_{k}, D_{i}=\bar{e}_{i s} S_{s}+\bar{\varepsilon}_{i j} E_{j}
$$

Now, we write Equation 8 with another subscripts $a, b, c$ and $d$ which can take only the values 1 and 3 . Therefore, Equation 8 may be written as:

$$
T_{a b}=\bar{c}_{a b c d} S_{c d}-\bar{e}_{k a b} E_{k}, \quad D_{i} \bar{e}_{i a b} S_{a b}+\bar{\varepsilon}_{i j} E_{j}
$$

Assume the semiconductor film with thickness $2 \mathrm{~h}$ and from the Equation 4 by integrating for $i=1,3$ regarding to $x_{2}$ within the film thickness. We may get the following equations of motion with the effect of rotation in two-dimensions, the law of Gauss and conservation of charge:

$$
\begin{aligned}
& T_{a b, a}+\frac{1}{2 h}\left[T_{2 h}\left(x_{2}=h\right)-T_{2 b}\left(x_{2}=-h\right)\right] \\
& =\rho\left(\ddot{u}_{b}-\Omega^{2}{ }_{u b}-2 \Omega \dot{u}_{a}\right) \\
& D_{a, a}+\frac{1}{2 h}\left[D_{2}\left(x_{2}=h\right)-D_{2}\right]\left(x_{2}=-h\right)=q n \\
& q \dot{n}+J_{a, a}+\frac{1}{2 h}\left[J_{2}\left(x_{2}=h\right)-J_{2}\left(x_{2}=-h\right)\right]
\end{aligned}
$$

where $u_{a}, T_{a b}, D_{a}, J_{a}$ and $n$ are averages of the quantities for the film thickness.

\section{Propagation of SH Waves}

As it is clear in Fig. 1, we consider the propagation of anti-plane SAW in piezoelectric dielectric semi-space covered by a non-piezoelectric semiconductor thin film of silicon (Abd-Alla et al., 2016). Assuming the direction propagation is $x_{1}$, then the component displacement and the electric potential which are effective in this case are:

$$
\begin{aligned}
& u_{1}=u_{2}=0, u_{3}^{p}=u_{3}^{p}\left(x_{1}, x_{2}, t\right), \\
& \varphi^{p}=\varphi\left(x_{1}, x_{2}, t\right)
\end{aligned}
$$

Therefore, the corresponding components of stain and electric field which are non-vanishing are:

$$
\left\{\begin{array}{l}
2 S_{13} \\
2 S_{23}
\end{array}\right\}=\nabla u_{3}^{p},\left\{\begin{array}{l}
E_{1} \\
E_{2}
\end{array}\right\}=-\nabla \phi^{p}
$$

where the quantities of the substrate are denoted by the superscript " $p$ " and $\nabla=i_{1} \partial_{1}+i_{2} \partial_{2}$ is denoted to the gradient operator in two-dimensions. Also, the components of stress and electric displacements that are nontrivial are:

$$
\left\{\begin{array}{l}
T_{13} \\
T_{23}
\end{array}\right\}=c_{44}^{p} \nabla u_{3}^{p}+e_{15}^{p} \nabla \phi^{p} .\left\{\begin{array}{l}
D_{1} \\
D_{2}
\end{array}\right\}=e_{15}^{p} \nabla u_{3}^{p}-\varepsilon_{11}^{p} \phi^{p}
$$

Now, the equations of motion and charge are:

$c_{44}^{p} \nabla^{2} u_{3}^{p}+e_{15}^{p} \nabla^{2} \phi^{p}=\rho^{p} \ddot{u}_{3}^{p}, e_{15}^{p} \nabla^{2} u_{3}^{p}-\varepsilon_{11}^{p} \nabla^{2} \phi^{p}=0$

where, $\nabla^{2}$ is the two-dimensional Laplacian $\nabla^{2}=\partial_{1}^{2}+\partial_{2}^{2}$. Assuming (Abd-Alla and Asker, 2016):

$\psi^{p}=\varphi^{p}-\frac{e_{15}^{p}}{\varepsilon_{11}^{p}} u_{3}^{p}$

then:

$\begin{array}{ll}T_{23}^{p}=c_{44}^{p^{*}} u_{3,2}^{p}+e_{15}^{p} \psi_{, 2}^{p}, & T_{31}^{p}=c_{44}^{p^{*}} u_{3,1}^{p}+e_{15}^{p} \psi_{, 1}^{p} \\ D_{1}^{p}=-\varepsilon_{11}^{p} \psi_{, 1}^{p}, & D_{2}^{p}=-\varepsilon_{11}^{p} \psi_{, 2}^{p}\end{array}$

Which lead to write the Equation 16 as:

$c_{44}^{p^{*}} \nabla^{2} u_{3}^{p}=\rho^{p} \ddot{u}_{3}^{p}, \quad \nabla^{2} \psi^{p}=0$

where:

$c_{44}^{p^{*}}=c_{44}^{p}+\frac{\left(e_{15}^{p}\right)^{2}}{\varepsilon_{11}^{p}}=c_{44}^{p}\left[1+\left(k_{15}^{p}\right)^{2}\right],\left(k_{15}^{p}\right)^{2}=\frac{\left(e_{15}^{p}\right)^{2}}{c_{44}^{p} \varepsilon_{11}^{p}}$

The solutions of Equation 19 should satisfy:

$u_{3}^{p}, \phi^{p} \rightarrow 0, x_{2} \rightarrow+\infty$

which are so-called the radiation conditions. Considered the solutions of Equation 19 as:

$$
\begin{aligned}
& u_{3}^{p}, A \exp \left(-\xi_{2} x_{2}\right)\left\{\exp i\left(\xi_{1} x_{1}-\omega t\right)\right\}, \psi^{p} \\
& =B \exp \left(-\xi_{1} x_{2}\right)\left\{\exp i\left(\xi_{1} x_{1}-\omega t\right)\right\}
\end{aligned}
$$

with $A$ and $B$ are constants should be obtained and $\xi_{2}$ must be greater than zero for decaying comportment far from the surface. It is easy to see that Equation $22_{2}$ already satisfies $(19)_{2}$. In order Equation $22_{1}$ satisfy Equation $19_{1}$ we must have:

$c_{44}^{p^{*}}\left(\xi_{1}^{2}-\xi_{2}^{2}\right)=\rho^{p} \omega^{2}$

Thus, $\xi_{2}$ may be written as:

$\xi_{2}^{2}=\xi_{1}^{2}-\frac{\rho^{p} \omega^{2}}{c_{44}^{p^{*}}}=\xi_{1}^{2}\left(1-\frac{v^{2}}{v_{T}^{2}}\right)>0$ 
where:

$$
v^{2}=\frac{\omega^{2}}{\xi_{1}^{2}}, \quad v_{T}^{2}=\frac{c_{44}^{p^{*}}}{\rho^{p}}
$$

For using boundary and continuity conditions, one may use the following:

$$
\begin{aligned}
\varphi^{p} & =\left[B \exp \left(-\xi_{1} x_{2}\right)+\frac{e_{15}^{p}}{\varepsilon_{11}^{p}} A \exp \left(-\xi_{2} x_{2}\right)\right] \exp \left[i\left(\xi_{1} x_{1}-\omega t\right)\right], \\
T_{23}^{p} & =-\left[\begin{array}{l}
A c_{44}^{p^{*}} \xi_{2} \exp \left(-\xi_{2} x_{2}\right) \\
+e_{15}^{p} B \xi_{1} \exp \left(-\xi_{1} x_{2}\right)
\end{array}\right] \exp \left[i\left(\xi_{1} x_{1}-\omega t\right)\right] \\
D_{2}^{p} & =\varepsilon_{11}^{p} B \xi_{1} \exp \left(-\xi_{1} x_{2}\right) \exp \left[i\left(\xi_{1} x_{2}-\omega t\right)\right]
\end{aligned}
$$

First, we try to solve the problem for the part of the vacuum.

Therefore, the Laplace equation must be satisfied for electric potential $\varphi^{0}$ in the free surface:

$$
\begin{aligned}
& x_{2}<0 \\
& \nabla^{2} \varphi^{0}=0, x_{2}<0, \varphi^{0} \rightarrow 0, x_{2} \rightarrow-\infty
\end{aligned}
$$

and the solutions of the Equation 27 are given as follows:

$$
\varphi^{0}=C \exp \left(\xi_{1} x_{2}\right) \exp \left[i\left(\xi_{1} x_{1}-\omega t\right)\right]
$$

where, $C$ is an unknown must be determined. Thus, from Equation 28 in the vacuum, yields:

$$
D_{2}^{0}=-\varepsilon_{0} \xi_{1} C \exp \left(\xi_{1} x_{2}\right) \exp \left[i\left(\xi_{1} x_{1}-\omega t\right)\right]
$$

Next, we need to find the solution of the semiconductor with rotation.

In the $x_{1}$-direction, we assume that DC is a biasing electric field. Also, the semiconductor layer with $n=$ $n\left(x_{1}, t\right)$ is considered as one dimension problem. Then, we consider:

$$
\begin{aligned}
& u_{3}=A \exp \left[i\left(\xi_{1} x_{1}-\omega t\right)\right], \\
& \varphi=C \exp \left[i\left(\xi_{1} x_{1}-\omega t\right)\right], \\
& n=N \exp \left[i\left(\xi_{1} x_{1}-\omega t\right)\right]
\end{aligned}
$$

where, $N$ is unknown constant must be determined. It is easy to see that Equation 30 satisfies the conditions of the continuity for the components of the displacement the electric potential between the substrate semi-space and the layer. We utilize a prime to denote the dielectric and elastic constants as well as the mass density of the film.
In the semiconductor layer, we used silicon that is considered as a cubic symmetry of crystal which does not have the piezoelectric coupling. Moreover, the stiffness elastic constants and dielectric constants are the same as Equation 31 in (Abd-Alla et al., 2016).

From Equation 9 and 5, we get:

$$
\begin{aligned}
T_{13}=\bar{c}_{44}^{\prime} S_{13}=\bar{c}_{44}^{\prime} u_{3,1}=\bar{c}_{44}^{\prime} i \xi_{1} A \exp \left[i\left(\xi_{1} x_{1}-\omega t\right),\right. \\
D_{1}=\bar{\varepsilon}_{11}^{\prime} E_{, 1}=-\bar{\varepsilon}_{11}^{\prime} \phi_{11}=-\bar{\varepsilon}_{11}^{\prime} i \xi_{1} C \exp \left[i\left(\xi_{1} x_{1}-\omega t\right)\right] \\
J_{1}=-q \bar{n} \mu_{11} \phi_{, 1}+q n \mu_{11} \bar{E}_{1}-q d_{11} n_{, 1} \\
=\left(-q \bar{n} \mu_{11} i \xi_{1} C+q N \mu_{11} \bar{E}_{1}-q d_{11} i \xi_{1} N\right) \times \\
\quad \exp \left[i\left(\xi_{1} x_{1}-\omega t\right) .\right.
\end{aligned}
$$

\section{Continuity Conditions and Frequency Equation}

According to continuity conditions of the component of electric potential between the piezoelectric semi-space and the layer of silicon and boundary condition's yields the flowing equations:

- From the continuity Equation $\varphi^{p}=\varphi^{0}$ at $x_{2}=0$ and using the Equation 26 and 28, we may get:

$$
B+\frac{e_{15}^{p}}{\varepsilon_{11}^{p}} A=C
$$

- Substitution the Equation $31_{1}$ and $26_{2}$ into Equation 10, we obtain:

$$
\bar{c}_{44}^{\prime} \xi_{1}^{2} A+\frac{1}{2 h}\left(A c_{44}^{p^{*}} \xi_{2}+e_{15}^{p} B \xi_{1}\right)=\rho^{\prime}\left(\omega^{2}-\Omega^{2}\right) A
$$

- Substitution the Equation 26, 29 and 31 into Equation 11, we get:

$$
\varepsilon_{11}^{\prime} \xi_{1}^{2} C+\frac{1}{2 h}\left(\varepsilon_{11}^{p} \xi_{1} B+\varepsilon_{0} \xi_{1} C\right)=q N
$$

- Substitution the Equation 30 and $31_{3}$ into Equation 12, we have:

$$
-q i \omega N+i \xi_{1}\left(-q \bar{n} \mu_{11} i \xi_{1} C+q N \mu_{11} \bar{E}_{1}-q d_{11} i \xi_{1} N\right)=0
$$

The system of Equation 32-35 are linear and homogeneous for the constants $A, B, C$ and $N$. The determinant of the coefficient matrix has to vanish to get nontrivial solutions, which imply:

$\left|a_{i j}\right|=0, \quad i, j=1,2,3,4$

where: 


$$
\begin{aligned}
& a_{11}=\frac{e_{15}^{p}}{\varepsilon_{11}^{p}}, a_{12}=1, a_{13}=-1, a_{14}=0, \\
& a_{21}=\left(\rho^{\prime}\left(\omega^{2}-\Omega^{2}\right)-\bar{c}_{44}^{\prime} \xi_{1}^{2}-\frac{c_{44}^{p^{*}} \xi_{2}}{2 h}\right), \\
& a_{22}=-\frac{e_{15}^{p} \xi_{1}}{2 h}, a_{23}=0, a_{24}=0, a_{31}=0, \\
& a_{32}=\frac{\varepsilon_{11}^{P} \xi_{1}}{2 h}, a_{33}=\frac{\varepsilon_{0} \xi_{1}}{2 h}+\bar{\varepsilon}_{11}^{\prime} \xi_{1}^{2}, a_{34}=-q, \\
& a_{41}=0, a_{42}=0, a_{43}=q \bar{n} \mu_{11} \xi_{1}^{2}, \\
& a_{44}=-q i \omega+i \xi_{1} q \mu_{11} \bar{E}_{1}+q d_{11} \xi_{1}^{2} .
\end{aligned}
$$

The solution of Equation 36 gives a relation between $\omega$ and $\xi_{1}$ that is so-called the frequency equation or the dispersion relation of the surface acoustic wave.

Now, we obtain the frequency equation of surface acoustic waves in two cases depends on the boundary condition at the free surface as follows.

\section{The Open Condition at the Free Surface}

In this case the surface acoustic wave velocity $v=$ $\omega / \xi_{1}$, Equation 37 may be expressed as:

$$
\left(\frac{v^{2}-\Omega^{*_{2}}}{v_{T}^{\prime 2}}-1\right) \frac{2 \bar{c}_{44}^{\prime} h \xi_{1}}{c_{44}^{p^{*}}}-\sqrt{1-\frac{v^{2}-\Omega^{* 2}}{v_{T}^{2}}}+\left(\bar{K}_{15}\right)^{2}=\frac{\left(\bar{K}_{15}\right)^{2}}{\epsilon_{1}}
$$

where $\epsilon_{1}=1+\frac{\varepsilon_{0}}{\varepsilon_{11}^{p}}+\frac{2 \bar{\varepsilon}_{11}^{\prime} h \xi_{1}}{\varepsilon_{11}^{p}}+\frac{2 q \bar{n} \mu_{11} h}{\varepsilon_{11}^{p}\left[d_{11} \xi_{1}+i\left(\mu_{11} \bar{E}_{1}-v\right)\right]}$ and it is used some dimensionless quantities as:

$$
\Omega^{*}=\frac{\Omega}{\xi_{1}}, v^{2}=\frac{\omega}{\xi_{1}}, v_{T}^{\prime 2}=\frac{\bar{c}_{44}^{\prime}}{\rho^{\prime}},\left(\bar{K}_{15}\right)^{2}=\frac{\left(e_{15}^{p}\right)}{\varepsilon_{11}^{p} c_{44}^{p^{*}}}
$$

If $h=0$, i.e., the semiconductor layer does not exist, we obtain that the velocity $v_{B-G}$ of Bleustein-Gulyaev wave (Bleustein, 1968; Gulyaev, 1969):

$$
v=v_{T}^{2}\left(1-\frac{\left(\bar{K}_{15}\right)^{4}}{\left(1+\varepsilon_{11}^{p} / \varepsilon_{0}\right)^{2}}\right)+\Omega^{*_{2}}=v_{B-G}^{2}
$$

If $\bar{K}_{15}=0$, in this case, we obtain the special case which is known as the wave is purely elastic medium and it has:

$$
\left(\frac{v^{2}-\Omega^{*_{2}}}{v_{T}^{\prime 2}}-1\right) \frac{2 \bar{c}_{44}^{\prime} h \xi_{1}}{c_{44}^{p^{*}} \quad 1}-\sqrt{1-\frac{v^{2}}{v_{T}^{2}}}=0
$$

From Equation 40, it is easy to calculate the wave velocity (Love wave (Yang, 2008)).
In the case of the imaginary part of the complex wave velocity may change its sign, i.e., transition from a damped to a growing wave, so that:

$v=\frac{\omega}{\xi_{1}}=\mu_{11} \bar{E}_{1}$

This means that, the surface acoustic wave is equal to the carrier drift velocity.

The Short Condition at the Free Surface (at $\varepsilon_{0}=0$ )

In this case, the electrically short condition yields:

$\bar{\varepsilon}_{11}^{\prime} \xi_{1}^{2} C+\frac{1}{2 h}\left(\varepsilon_{11}^{p} \xi_{1} B\right)=q N$

and the frequency equation of surface acoustic waves may be given by:

$\left(\frac{v-\Omega^{* 2}}{v_{T}^{\prime 2}}-1\right) \frac{2 \bar{c}_{44}^{\prime} h \xi_{1}}{c_{44}^{p^{*}}}-\sqrt{1-\frac{v^{2}-\Omega^{* 2}}{v_{T}^{2}}}+\left(\bar{K}_{15}\right)^{2}=\frac{\left(\bar{K}_{15}\right)^{2}}{\epsilon_{2}}$

where:

$$
\epsilon_{2}=1+\frac{\bar{\varepsilon}_{11}^{\prime}}{\varepsilon_{11}^{p}}+\frac{2 q \bar{n} \mu_{11} h}{\varepsilon_{11}^{p}\left[d_{11} \xi_{1}+i\left(\mu_{11} \bar{E}_{1}-v\right)\right]}
$$

In this case the velocity $v_{B-G}$ of Bleustein-Gulyaev wave is given by:

$v^{2}=v_{T}^{2}\left[1-\left(\bar{K}_{15}\right)^{4}\right]+\Omega^{* 2}=v_{B-G}^{2}$

\section{Numerical Results}

Now, we calculate the zero order of the velocity denoted by $v_{(0)}$ when neglecting the semiconductor. Then the Equation 38 becomes:

$$
\left(\frac{v_{(0)}^{2}-\Omega^{*_{2}}}{v_{T}^{\prime 2}}-1\right) \frac{2 \bar{c}_{44}^{\prime} h \xi_{1}}{c_{44}^{p^{*}}}-\sqrt{1-\frac{v_{(0)}^{2}-\Omega^{* 2}}{v_{T}^{2}}}+\bar{K}_{15}^{2}=\frac{\bar{K}_{15}^{2}}{\epsilon_{3}}
$$

where:

$$
\epsilon_{3}=1+\frac{\varepsilon_{0}}{\varepsilon_{11}^{p}}+\frac{\varepsilon_{11}^{\prime}}{\varepsilon_{11}^{p}}
$$

To get the first order of the velocity $v_{(1)}$, we replace $v_{(0)}$ into Equation 37, Yields: 


$$
\left(\frac{v_{(1)}^{2}-\Omega^{*_{2}}}{v_{T}^{\prime 2}}-1\right)\left(\frac{2 \bar{c}_{44}^{\prime} h \xi_{1}}{c_{44}^{p^{*}}}\right)-\sqrt{1-\frac{v_{(1)}^{2}-\Omega^{* 2}}{v_{T}^{2}}}+\bar{K}_{15}^{2}=\frac{\bar{K}_{15}^{2}}{\epsilon_{4}}
$$

where:

$$
\epsilon_{4}=1+\frac{\varepsilon_{0}}{\varepsilon_{11}^{p}}+\frac{2 \varepsilon_{11}^{\prime} h \xi_{1}}{\varepsilon_{11}^{p}}+\frac{2 q \bar{n} \mu_{11} h}{\varepsilon_{11}^{p}\left[d_{11} \xi_{1}+i\left(\mu_{11} \bar{E}-v_{(0)}^{2}\right)\right]}
$$

For the PZT-5H piezoelectric materials, Abd-Alla and Asker (2016), with:

$$
\begin{aligned}
& c_{44}^{p}=2.3 \times 10^{10} \mathrm{~N} / \mathrm{m}^{2}, \varepsilon_{11}^{p}=1700 \varepsilon_{0}, \\
& \varepsilon_{0}=8.854 \times 10^{12} \text { farads } / \mathrm{m}, \\
& e_{15}^{p}=17.0, \quad \rho_{0}=7500 \mathrm{~kg} / \mathrm{m}^{3}
\end{aligned}
$$

For silicon with, Lide (2001):

$$
\rho_{0}^{\prime}=2332 \mathrm{~kg} / \mathrm{m}^{3}, c_{44}^{\prime}=7.956 \times 10^{10} \mathrm{~N} / \mathrm{m}^{2}, \varepsilon_{11}^{\prime}=11.8 \varepsilon_{0}
$$

where at $300^{\circ} \mathrm{K}$, the mobility of electrons and holes of silicon is:

$$
\mu_{n}=1500 \mathrm{~cm}^{2} / V-\mathrm{sec}, \mu_{p}=480 \mathrm{~cm}^{2} / V-\mathrm{sec}
$$

Figure 2-5 show $Y_{(0)}$ and $Y_{(1)}$ versus $X$, where $X$ is dimensionless wave number, $Y_{(0)}$ and $Y_{(1)}$ are the dimensionless velocities for zero and first order, respectively which are defined by:

$$
X=\frac{2 h \xi_{1}}{\pi}, Y_{(0)}=\frac{v_{(0)}}{v_{B-G}}, Y_{(1)}=\frac{\operatorname{Re}\left\{v_{(1)}\right\}}{v_{B-G}}
$$

where, $\gamma$ is a without dimension number considered by:

$$
\gamma=\frac{\mu_{11} \bar{E}_{1}}{v_{B-G}}
$$

The phase velocity computation; at zero and first order; for the short and open electric cases versus wave number when change rotation at $\gamma=5$ and $h=$ 0.005 are illustrated in Fig. 2 to 5 . It is seen that $Y_{0}$ and $Y_{1}$ decrease when increasing the wave number $X$. However, they increase when increasing the values of the rotation $\Omega$.

Figure 6 displays the comparison between the dispersion relations of zero-order $Y_{0}$ versus the dimensionless wave number $X$, respectively for electrically open and short conditions. While, Fig. 7 illustrates the comparison between $Y 1$ as function of $X$, respectively for short and open electric conditions. It is clear that the values of $Y_{0}$ and $Y_{1}$ as function of $X$ are greater in the short electric case than the open electric case when the value of rotation $\Omega=10$.

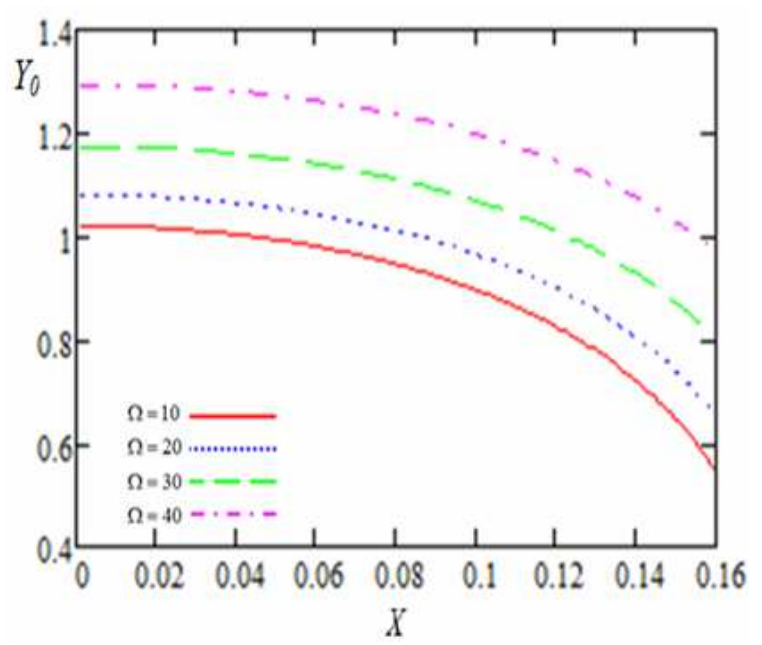

Fig. 2. The dispersion relations of the zero-order $Y_{0}$ against $X$ for various values of $\Omega$ (open electric case).

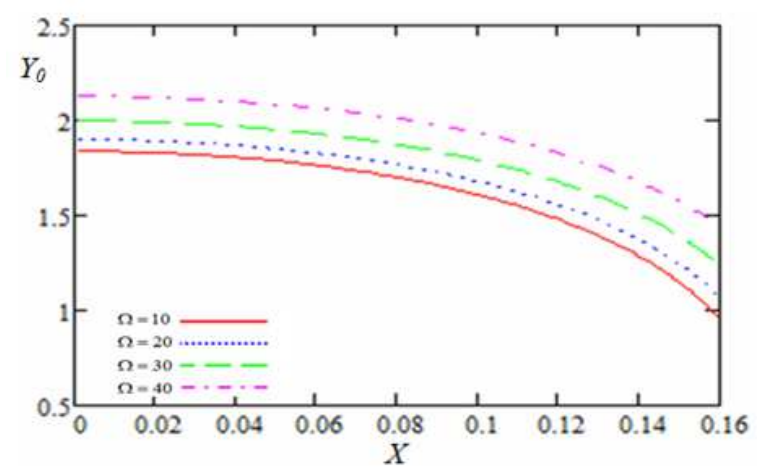

Fig. 3. The dispersion relations of the zero-order $Y_{0}$ against $X$ for various values of $\Omega$ (short electric case).

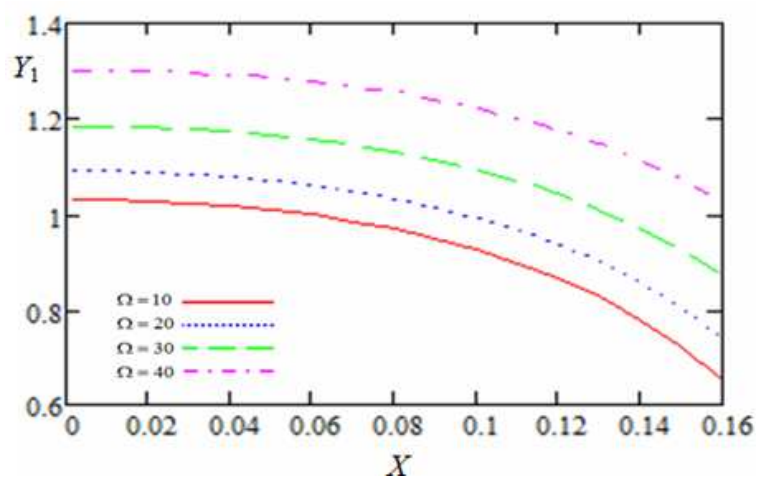

Fig. 4. The dispersion relations of the zero-order $Y_{1}$ against $X$ for various values of $\Omega$ (open electric case) 


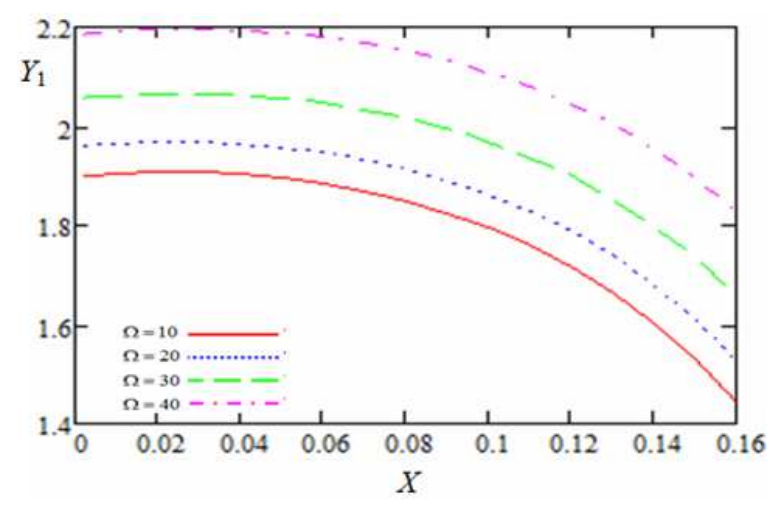

Fig. 5. The dispersion relations of the zero-order $Y_{1}$ against $X$ for various values of $\Omega$ (short electric case)

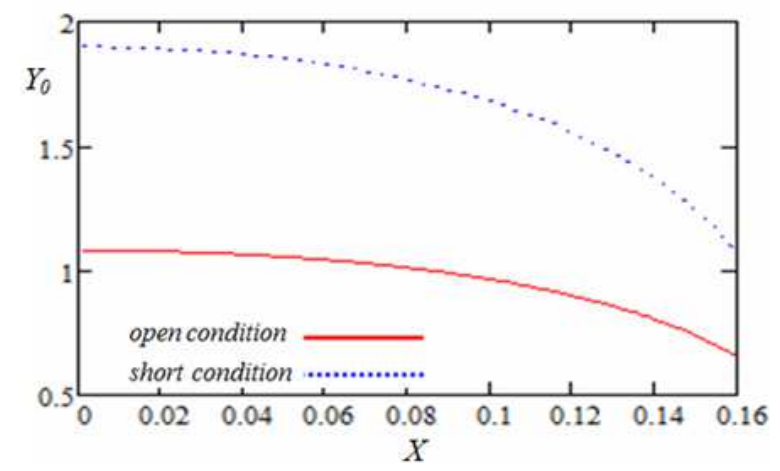

Fig. 6. The comparison between dispersion relations of the zeroorder $Y_{0}$ at $\Omega=10$ against $X$ when open and short cases

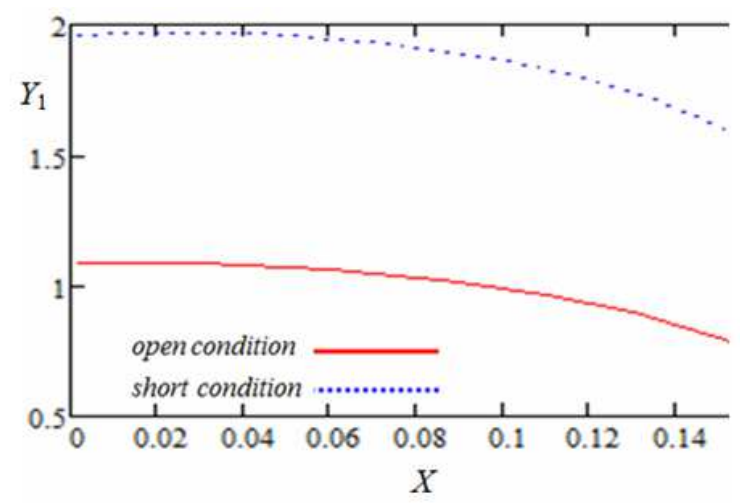

Fig. 7. The comparison between dispersion relations of the zeroorder $Y_{1}$ at $\Omega=10$ against $X$ when open and short cases

\section{Conclusion}

We show that a SH of SAW exist in a piezoelectric material semi-space covered by of a semiconducting film and we obtain a mathematical model of a system of equations in two dimensions for the motion of a thin membrane of piezoelectric semiconductors to describe the semiconductor film. This problem generalizes what is so-called Bleustein-Gulyaev wave in a semi-space of non-conducting piezoelectric ceramics. Furthermore, the influence of the rotation and the semi-conduction effect on the wave velocity causes both wave attenuation and dissipation.

\section{Acknowledgment}

The author is grateful to the reviewers and the editors for their helpful suggestions that improved this article.

\section{Ethics}

This article is original and contains unpublished material. The corresponding author confirms that all of the other authors have read and approved the manuscript and no ethical issues involved.

\section{References}

Abd-Alla, A.N. and F. Alshaikh, 2016. The influence of piezoelectric and electromagnetic effects on the propagation of rayleigh surface waves in smart material. J. Comput. Theoretical Nanosci., 13: 8674-8682. DOI: $10.1166 /$ jetn.2016.6029

Abd-Alla, A.N. and G.A. Maugin, 1987. Nonlinear magnetoacoustic equations. J. Acoustical Society Am., 82: 1746-1752. DOI: 10.1121/1.395167

Abd-Alla, A.N. and G.A. Maugin, 1990a. Nonlinear equations for thermoelastic magnetizable conductors. Int. J. Eng. Sci., 28: 589-603. DOI: 10.1016/0020-7225(90)90088-Z

Abd-Alla, A.N. and G.A. Maugin, 1990b. Linear and nonlinear surface waves on magnetostrictive substrates. Eur. J. Mechan., 9: 313-340.

Abd-Alla, A.N. and N. Asker, 2016. Numerical simulations for the phase velocities and the electromechanical coupling factor of the BleusteinGulyaev waves in some piezoelectric smart materials. Math. Mechan. Solids, 21: 539-551.

DOI: $10.1177 / 1081286514527861$

Abd-Alla, A.N., 1999. Nonlinear constitutive equations for thermo-electroelastic materials. Mechan. Res. Commun., 24: 335-346. DOI: 10.1016/S0093-6413(99)00032-4

Abd-Alla, A.N., N.F. Hasbullah and H.M. Hossen, 2016. The frequency equations for shear horizontal waves in semiconductor/piezoelectric structures under the influence of initial stress. J. Comput. Theoretical Nanosci., 13: 6475-6481.

DOI: $10.1166 /$ jctn.2016.5589

Bleustein, J.L., 1968. A new surface wave in piezoelectric materials. Applied Phys. Lett., 13: 412-413. DOI: $10.1063 / 1.1652495$ 
Gua, C. and E. Jin, 2015. Shear-horizontal surface waves in a half-space of piezoelectric semiconductors. Philosophical Magaz. Lett., 95: 92-100. DOI: $10.1080 / 09500839.2015 .1011249$

Gulyaev, V.Y., 1969. Electroacoustic surface waves in solids. J. Exp. Theoretical Phys. Lett., 9: 37-38.

Hutson, A.R. and D.L. White, 1962. Elastic wave propagation in piezoelectric semiconductors. J. Applied Phys., 33: 40-47. DOI: 10.1063/1.1728525

Kielczynski, P.J., W. Pajewski and M. Szalewski, 1989. Shear-horizontal surface waves on piezoelectric ceramics with depolarized surface layer. IEEE Trans. Ultrason. Ferroelectr. Freq. Control, 36: 287-293. DOI: 10.1109/58.19163

Lide, D.R., 2001. RC Handbook of Chemistry and Physics. 82nd Edn., CRC Press, Cleveland.

Liu, J., W. Wei and D. Fang, 2010. Propagation behaviors of shear horizontal waves in piezoelectricpiezomagnetic periodically layered structures. Acta Mech. Solida Sin., 23: 77-84.

DOI: 10.1016/S0894-9166(10)60009-2

Piliposian, G.T., A.S. Avetisyan and K.B. Ghazaryan, 2012. Shear wave propagation in periodic phononic/photonic piezoelectric medium. Wave Mot., 49: 125-134. DOI: 10.1016/j.wavemoti.2011.08.001

Royer, D. and E. Dieulesaint, 2000. Elastic Waves in Solids I: Free and Guided Propagation. 1st Edn., Springer-Verlag, Berlin, Germany, ISBN-10: 3540659323, pp: 374.

Son, M.S. and Y.J. Kang, 2011. Propagation behavior of $\mathrm{SH}$ waves in layered piezoelectric plates. J. Mechanical Sci. Technol., 25: 613-619. DOI: $10.1007 / \mathrm{s} 12206-011-0114-8$

Tiersten, H.F., D.S. Stevens and P.K. Das, 1980. Acoustic surface wave accelerometer and rotation rate sensor. Proceedings of the IEEE- International Ultrasonics Symposium, Nov. 5-7, IEEE Xplore Press, pp: 692-695. DOI: 10.1109/ULTSYM.1980.197488

Topolov, V.Y. and C.R. Bowen, 2008. From Smart Materials to Piezo-Composites. In: Electromechanical Properties in Composite Based on Ferroelectrics, Topolov, V.Y. and C.R. Bowen (Eds.), Springer Science and Business Media, London, ISBN-10: 1848820003, pp: 1-10.
Vashishth, A.K. and A. Dahiya, 2013. Shear waves in a piezoceramic layered structure. Acta Mech., 224: 727-744. DOI: 10.1007/s00707-012-0772-3

Wren, T. and J.S. Budness, 1987. Surface waves perturbed by rotation. J. Applied Mechan., 54: 464-468. DOI: $10.1115 / 1.3173043$

Yang, J., 2008. The Mechanics of Piezoelectric Structures. 1st Edn., World Scientific Publishing Co., Singapore.

Ye, Z.G., 2008. Handbook of Advanced Dielectric, Piezoelectric and Ferroelectric Materials: Synthesis, Properties and Applications. 1st Edn., Elsevier, Cambridge, ISBN-10: 1845694007, pp: 1096.

Zakharenko, A.A., 2013. Fundamental modes of new dispersive SH-waves in piezoelectromagnetic plate. PRAMANA J. Phys., 81: 819-827. DOI: $10.1007 / \mathrm{s} 12043-013-0609-1$

\section{Nomenclature}

$\tau_{i j}$ is the stress tensor,

$S_{i j}$ is the strain tensor,

$D_{i}$ is the electric displacement vector,

$E_{i}$ is the electric field vector,

$\rho$ is the mass density of the piezoelectric material, $\varphi$ is the electric potential,

$c_{i j k l}$ is the elastic constants tensor,

$e_{i j k}$ is the piezoelectric constants tensor,

$\varepsilon_{i j}$ is the dielectric constants tensor,

$N_{j}$ Is the carrier density gradient,

$d_{i j}$ is the carrier diffusion constants tensor,

$E_{i}$ is the uniform DC electric field vector,

$q$ is the carrier charge,

$n$ is the steady state carrier density,

$n$ is the carrier density,

$\mu_{i j}$ is the carrier mobility tensor,

$J_{i}$ is the electric current vector,

$u_{i}$ is the elastic displacement vector, 